\title{
Lösungsmöglichkeiten nach fehlgeschlagenen Osteosynthesen im Bereich des Unterarmschaftes
}

\author{
Sebastian Hullmann, Ralf Breuer, Andreas Dávid
}

\section{Zusammenfassung}

Komplikationen durch fehlgeschlagene Osteosynthesen sind im Bereich des Unterarmschaftes eher selten $\mathrm{zu}$ beobachten. Refrakturen und primär insuffiziente Osteosynthesen mit mangelnder Reposition und/oder Retention sind frühzeitig durch einen Korrektureingriff zu behandeln, um Fehlheilungen und den resultierenden Komplikationen vorzubeugen. Bei ausbleibender Konsolidierung der Frakturen nach Osteosynthese kann 4-8 Wochen abgewartet werden, ob sich eine Pseudoarthrose manifestiert oder eine chirurgische Intervention notwendig ist. Die Reosteosynthese muss erfolgen, sobald sich die Heilungsverzögerung abzeichnet. Nach primärer Diastase sollte allerdings eine rasche Entscheidung zur Operation angestrebt werden, da die Pseudarthrosengefahr besonders groß ist. Fehlstellungen sind sofort zu beheben, da die Umwendbewegung des Unterarmes behindert wird. Synostosen erfordern eine frühzeitige Resektion, um eine Schrumpfung der Membrana interossea zu vermeiden, die ebenfalls zu einer eingeschränkten Unterarmdrehung führt.

\section{Einleitung}

Unterarmfrakturen im Schaftbereich werden vorzugsweise mit Plattenosteosynthesen (3,5 LCDCP oder DCP Implantate) versorgt [1]. Wegen der notwendigen hohen Kompression auf den Bruchspalt werden außer bei langen Trümmerzonen Kompressionsosteosynthesen angewandt. Alternativ hierzu bietet sich auch die intramedulläre Marknagelung an. Als entscheidender Nachteil der Marknägel wird die unsichere Rotationsfestigkeit und die mangelnde Kompression auf den Bruchspalt diskutiert. Besonders bei einfachen Quer- und kurzen Schrägbrüchen droht nach Marknagelung wegen der Instabilität im Bruchspalt und der zu großen Bewegung eine Pseudarthrose. Die Pseudoarthrosenrate liegt nach plattenosteosynthetischer Versorgung bei ca. drei Prozent [1,2,3,4]. Die Marknagelosteosynthese hat aber eine tendenziell höhere Pseudarthrosenge-

OP-JOURNAL 2004; 20: 108-111

(c) Georg Thieme Verlag KG Stuttgart · New York fahr. Bei initialer Diastase im Bruchspalt kann eine Pseudarthrose entstehen. Hauptproblem ist die mögliche gegenseitige Sperrwirkung der beiden Knochen, die eine Kompression durch Muskelkontraktion verhindert. Gerade am Unterarm ist es daher bedeutsam, durch die Osteosynthese sofort eine Kompression auf den Bruchspalt zu erzielen. Durch funktionelle Beanspruchung kann, wie etwa an Ober- oder Unterschenkel durch Belastung, kaum Druck im Bruchspalt ausgeübt werden.

Bei kindlichen Frakturen erfolgt die Osteosynthese dagegen auch beim Bruch eines der beiden Knochen zumeist mittels minimalinvasiv eingebrachter, intramedullärer und elastischer Titanmarknägel wie zum Beispiel die Prévot-Nagelung. Hier ist das Pseudarthrosenrisiko gering. Selbst bei Versorgung eines Knochens bei intaktem zweiten ist im Kindesalter fast nie eine Heilungsverzögerung festzustellen.

Als weitere typische Komplikation der Unterarmschaftfrakturen nach osteosynthetischer Versorgung gilt die Refraktur nach Metallentfernung. Die Literaturangaben zur Häufigkeit reichen vereinzelt von null bis zu mehr als 20 Prozent in Abhängigkeit von der verwendeten Osteosynthese, des Zeitpunktes der Metallentfernung und des Frakturtypes [1,2,5,6].

Ein besonderes Problem kann die Entwicklung einer Synostose zwischen Radius und Ulna sein, die zu einer Aufhebung der Unterarmrotation führt. Sie ist häufiger im ellenbogennahen Unterarm zu beobachten als im Schaftbereich.

Da Korrektureingriffe am Unterarm komplikationsträchtig sind und nicht immer ein gutes Funktionsergebnis erzielt werden kann, müssen die primären Osteosynthesen mit besonderer Sorgfalt durchgeführt werden, um Fehlheilungen möglichst zu vermeiden.

Im Übrigen ist mit unbefriedigenden Therapieergebnissen oder Komplikationen zu rechnen falls Reposition und/oder Retention der Fraktur nur unzureichend erfolgt sind. Vor allem die Unterarmdrehfähigkeit wird eingeschränkt, wenn die anatomischen Achsen der beiden Knochen nicht wiederhergestellt werden können. Die nachfolgenden Beispiele sollen die typischen, oben genannten Komplikationen und mögliche Lösungswege zur Darstellung bringen.

\section{Pseudarthrose}

Pseudarthrosen an den Unterarmknochen sind meist Folge einer nicht ausreichenden Kompression auf den Bruchspalt oder einer primären Diastase

Einen typischen Verlauf der Ausbildung einer Pseudoarthrose nach Unterarmmarknagelung und Therapieerfolg nach Verfahrenswechsel zeigt Abb.1.

Auf den dargestellten postoperativen Röntgenaufnahmen zeigt sich ein weit einsehbarer Frakturspalt in beiden Ebenen - längengerechte Reposition aber fehlender Kompression. 
Der in den Röntgenaufnahmen 6 Wochen postoperativ (Abb.1 c) weiterhin gut einsehbare Frakturspalt und die fehlende Kallusbildung an der Ulna lassen bereits auf eine verzögerte Bruchheilung schließen. Dies bestätigt sich in den weiteren Verlaufskontrollen (Abb.1 d) die eine hypertrophe Pseudoarthrose zur Darstellung bringen. Der Radius hingegen zeigt eine deutliche Durchbauungstendenz.
Dieser Therapieverlauf erfordert einen Verfahrenswechsel im Sinne einer Reosteosynthese, welcher zumeist durch eine Spongiosaplastik ergänzt wird, wie unter Abb.1f dargestellt.

Die Reosteosynthese erfolgt durch eine Plattenosteosynthese der Ulna und interfragmentäre Zugschraube bei isolierter Pseudarthrose. Bei Heilungsverzögerung beider Knochen werden auch beide durch

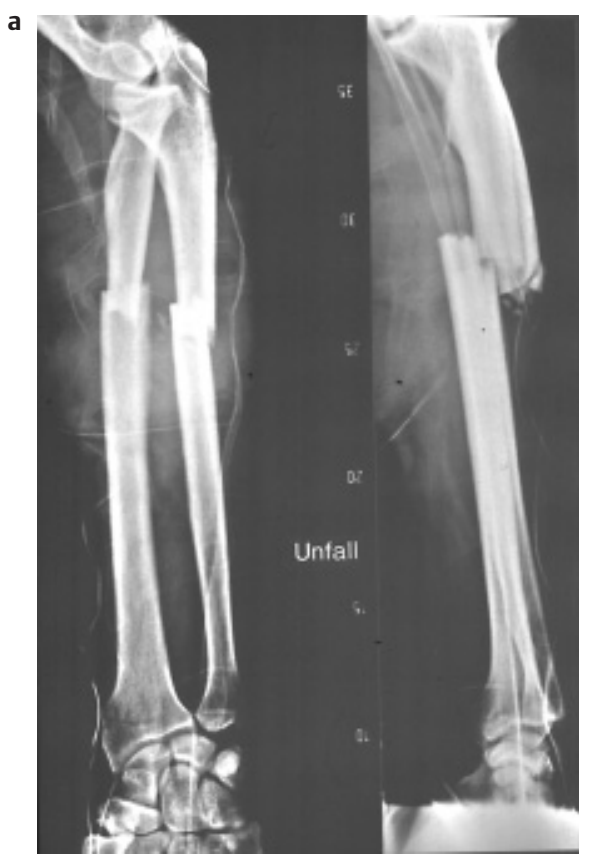

Abb.1a Dislozierte Unteramschaftfraktur.

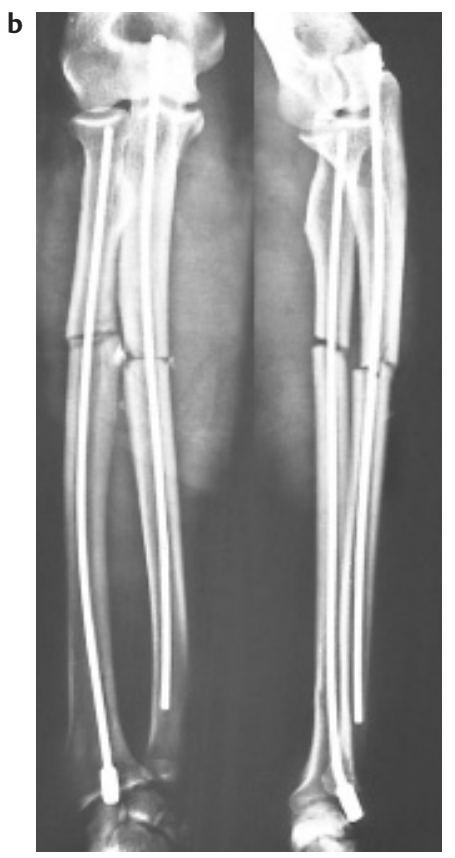

Abb.1b Nach intramedullärer Osteosynthese. eine Plattenosteosynthese versorgt. Es kann auf die Osteosynthese des 2.Knochens verzichtet werden, wenn schon eine weit gehende knöcherne Heilung $\mathrm{zu}$ beobachten ist (Abb.1). Die Abb.1f zeigt die vollständige knöcherne Durchbauung bei einem derartigen Vorgehen.

Eine autologe Spongiosaplastik ist nur bei hypotrophen Pseudarthrosen notwendig. Bei den hypertrophen Pseudoarthrosen wird auf eine Spongiosaplastik verzichtet. Durch Dekortikation oder Resektion des hypertrophen Kallus und Anlage an den Pseudarthrosenspalt kann die Knochenbruchheilung unterstützt werden, ohne dass eine Entnahme von Knochenmark an anderer Stelle notwendig wird.

Wird eine Spongiosaplastik durchgeführt, muss die Anlagerung exakt geplant werden. Die Spongiosa sollte keinesfalls auf die Membrana interossea aufgelegt werden, da eine Synostose droht.

Die Reosteosynthese wird sofort durchgeführt, wenn sich die Heilungsverzögerung radiologisch und klinisch bestätigt. Ein weiteres Zuwarten ist in der Regel erfolglos.
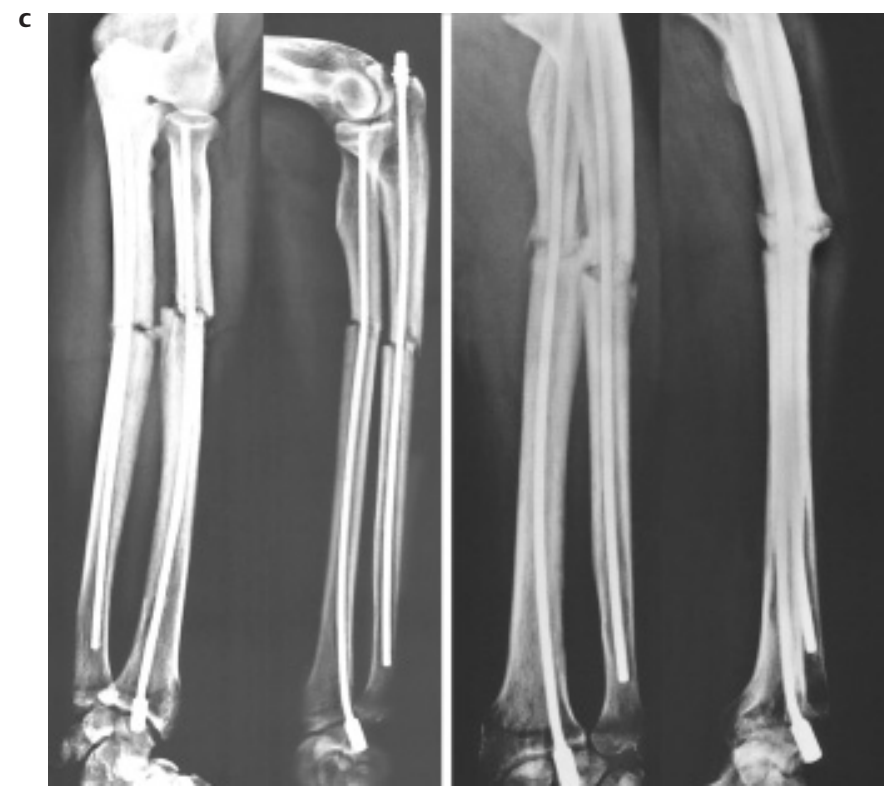

Abb.1cu.d 6 Wochen und 4 Monate postoperativ.

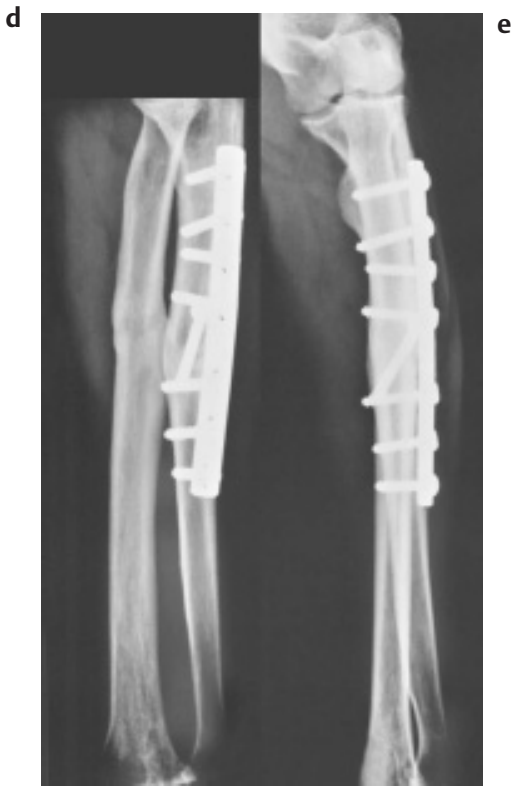

Abb.1e 1,5 Jahre nach Reosteosynthese, erfolgreiche knöcherne Ausheilung. 


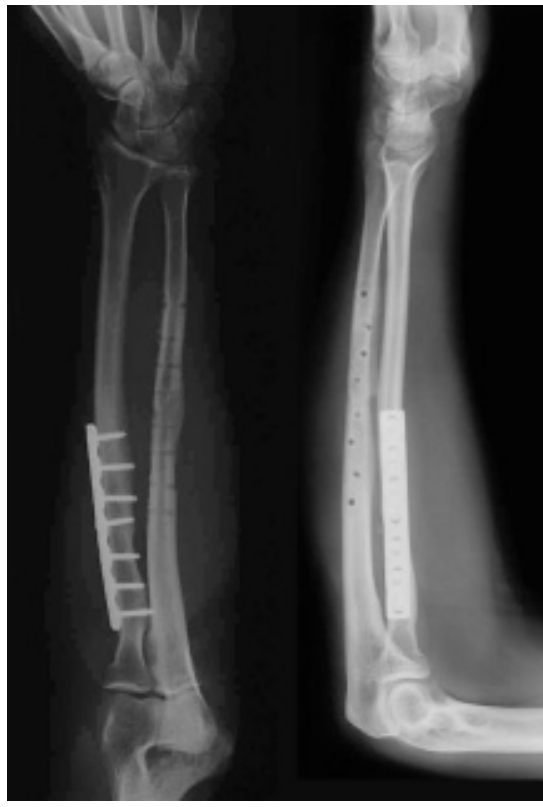

Abb. 2a Konsolidierte Unteramschaftfraktur. Postoperative Röntgenkontrolle nach Entfernung der ulnaren Plattenosteosynthese.

\section{Refraktur}

Hauptursache der Refraktur ist die vorzeitige Metallentfernung.

In der Regel kann auch eine Platte am Unterarmschaft belassen werden. Sie kann aber bei dorsaler Lage am Radius und an der Ulnakante stören, wenn nur eine geringe Weichteildeckung vorliegt. Gelegenlich verkleben auch Sehnen auf der Platte und behindern die Bewegung. Besonders häufig wird dies am distalen Radiusschaft beobachtet, wo die Sehnen der $\mathrm{Mm}$. extensor pollicis brevis und abduktor pollicis longus den Radiusschaft kreuzen. Marknägel werden meist entfernt, da sie an den Eintrittspunkten auftragen und daher die Haut und Sehnen irritieren können.

Eine weitere Ursache ist die Knochenatrophie, die sich unter einer Plattenosteosynthese entwickeln kann (Beispiel 2). Bei starker mechanischer Beanspruchung unmittelbar nach der Metallentfernung ist das Risiko einer Refraktur hoch. Eine Schonung von bis zu 3 Monaten nach Metallentfernung muss unter radiologischer Verlaufskontrolle dringend empfohlen werden. Viele Autoren empfehlen, die Platten an Radius und Ulna nacheinander zweizeitig zu entfer-

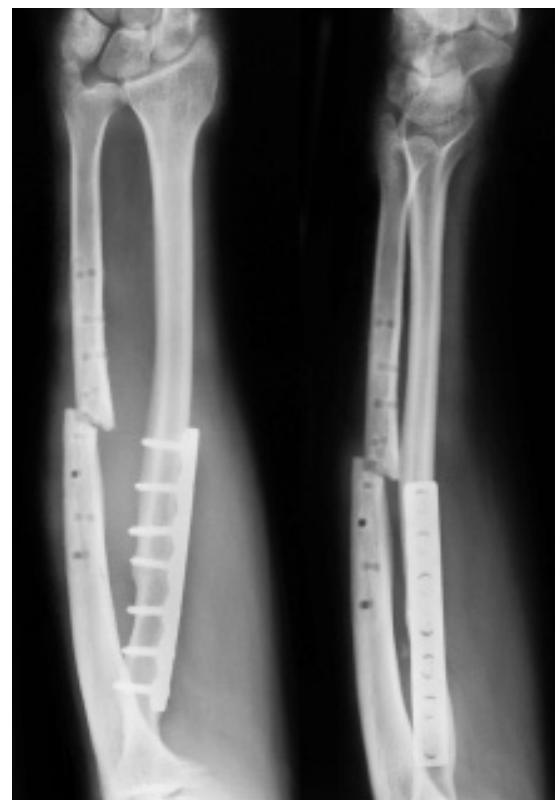

Abb. 2b Refraktur Ulnaschaft.

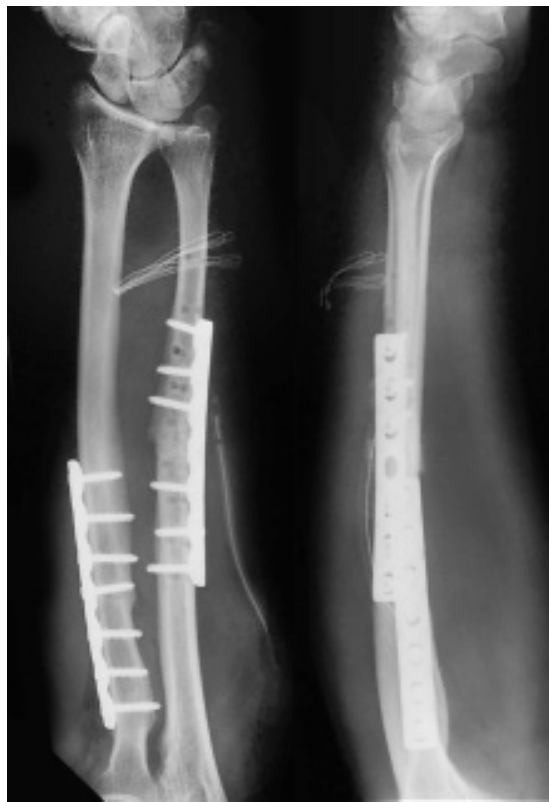

Abb. 2c Reosteosynthese Ulnaschaft.

nen. Bei atrophen Knochenheilungen ist dies sicher sinnvoll. Zeigen sich aber beide Unterarmknochen radiologisch sicher durchbaut, so kann die Implantatentfernung auch einzeitig vorgenommen werden.

Ein typisches Beispiel einer Refraktur nach primärer plattenosteosynthetischer Versorgung: bereits nach Entfernung der Ulnaplatte erkennt man die erhebliche Atrophie des Knochens (Abb.2).

Um einer Refraktur aufgrund der noch vorhandenen strukturellen Schwäche vorzubeugen, wurde nach Ausheilung zunächst nur eine der beiden Platten entfernt.

Trotz dieser Vorgehensweise kam es durch ein Bagatelltrauma zu einer ulnaren Refraktur (Abb.2 b).

Diese Komplikation zwingt zu einer erneuten operativen Intervention wie in diesem Beispiel durch eine Plattenreosteosynthese des Ulnaschaftes. Möglichst sollte eine Kompression durch Plattenspanngerät und Zugschraube (hier durch ein Plattenloch) angelegt werden.

Wie unter Abb.2c gezeigt, wurde die Platte am Radiusschaft belassen.

\section{Achsfehler}

Achsfehler führen zu einer eingeschränkten Rotation des Unterarmes

\section{Fehlstellung nach Osteosynthese einer} kindlichen Unterarmschaftfraktur (Abb. 3).

Die $\mathbf{A b b} \mathbf{3} \mathbf{3 b}$ zeigt die primäre osteosynthetische Versorgung der dislozierten Radiusschaftfraktur mit mangelhafter Reposition und fehlender Retention. Das wesentliche Problem eines Achsfehlers vor allem am Radius ist die Störung der Rotation.

Eine Fehlstellung muss sofort korrigiert werden. Ein Verfahrenswechsel ist nicht zwingend erforderlich. Bei einer Plattenosteosynthese kann die Platte am Radius belassen werden. Lediglich die Position der Platte auf einer Frakturseite (meist distal) muss geändert werden. Bei Marknagelosteosynthesen ist ein Verfahrenswechsel meist erforderlich. Dies gilt natürlich ganz besonders für die hoch instabilen K-Draht-Fixationen, wie die Abb. $3 \mathbf{c}$ u.d veranschaulichen. 
$a, b$
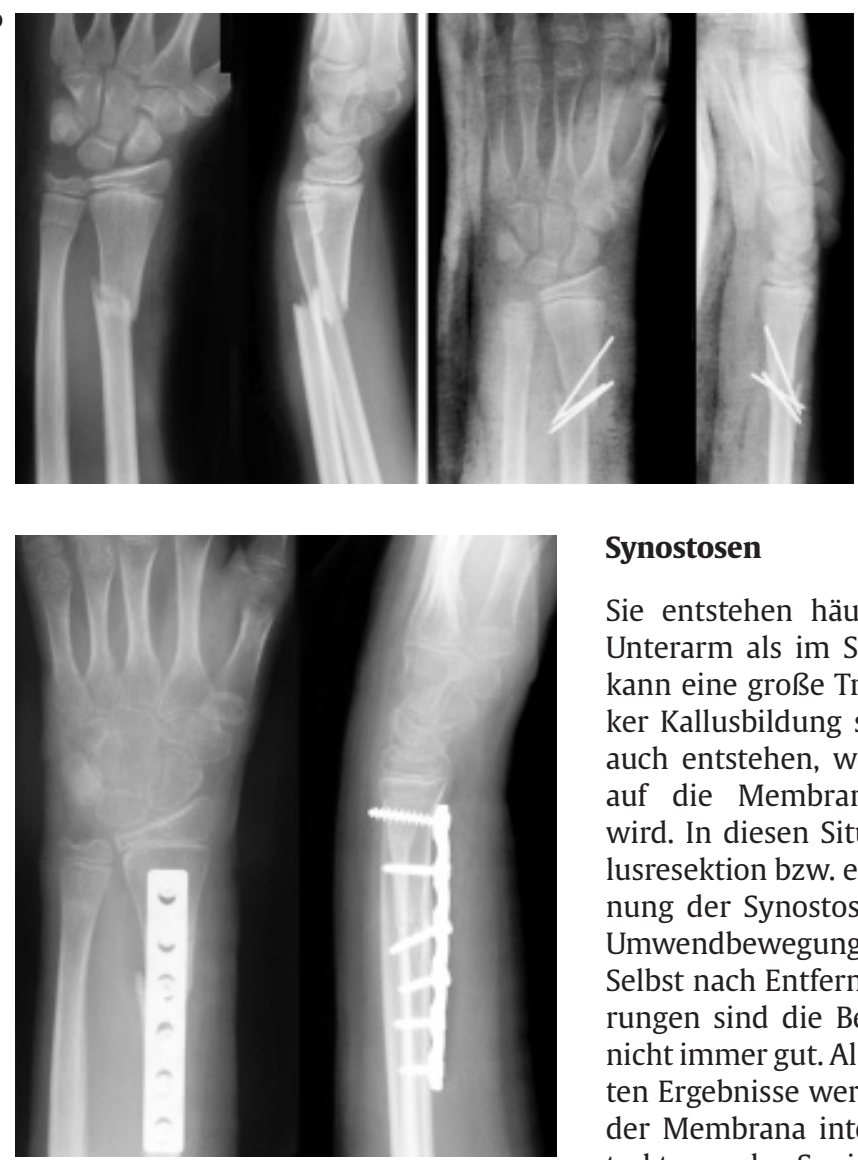

d

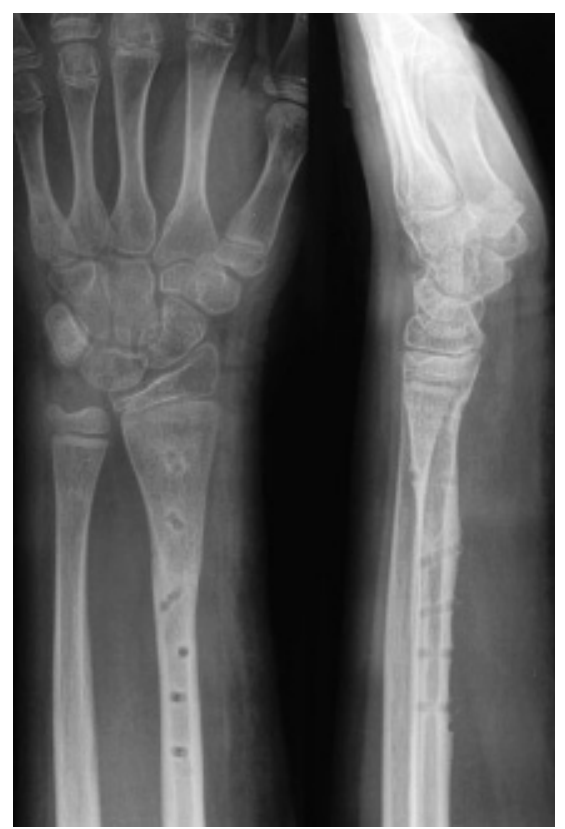

Abb. 3 Kindliche Unterarmschaftfraktur mit Dislokation des Radius (a), primärer Osteosynthese (b).

\section{Synostosen}

Sie entstehen häufiger am proximalen Unterarm als im Schaftbereich. Ursache kann eine große Trümmerzone mit starker Kallusbildung sein. Sie können aber auch entstehen, wenn Spongiosa direkt auf die Membrana interossea gelegt wird. In diesen Situationen ist eine Kallusresektion bzw. eine frühzeitige Entfernung der Synostose notwendig, um die Umwendbewegung wiederherzustellen. Selbst nach Entfernung der Überknöcherungen sind die Behandlungsergebnisse nicht immer gut. Als Ursache der schlechten Ergebnisse werden die Schrumpfung der Membrana interossea und die Kontrakturen der Supinatoren und Pronatoren diskutiert. Daher müssen derartige Synostosen bei den primären Osteosynthesen unbedingt vermieden werden.

\section{Literatur}

1 Chapmann MW, Gordon JE, Zissimos AG. Compression-plate fixation of acute fractures of diaphysis of the radius and ulna. JBJS Am 1989; 70: 1372

2 Hertel R, Psan M, Lambert S, Ballmer FT. Plate osteosynthesis of diaphyseal fractures of the redius and ulna. Injury 1996; 27: $545-$ 548

3 Oestern HJ, Tscherne H. Ergebnisse der AOSammelstudie über Unterarmschaftfrakturen. Unfallchirurg 1983; 86: 136

${ }^{4}$ Winckler S, Brug E, Baranowski D. Bündelnagelung bei Unterarmfrakturen. Indikation und Ergebnisse. Unfallchirurg 1991; 94 $335-341$

${ }^{5}$ Labosky DA, Cermak MB, Waggy CA. Forearm fractures plate:to remove or not to remove. J Hand Surg (Am) 1990; 15: 294 - 301

${ }^{6}$ Rosson JW, Shearer JR. Refractures of the removal of plates from the forearm. An avoidable complication. JBJS (Br) 1991; 73: $415-$ 417

Sebastian Hullmann

Assistenzarzt

Dr. med. Ralf Breuer

Assistenzarzt

Prof. Dr. med. Andreas Dávid

Klinkdirektor

Klink für Unfall- und Wieder-

herstellungschirurgie

Helios-Klinikum Wuppertal

Universitätsklinikum der Universität

Witten/Herdecke

Heusnerstraße 40

D-42283 Wuppertal 